\title{
Approach to increase flexibility in automobile body shops through component-integrated fixture functions
}

\author{
Achim K ampker ${ }^{1}$, Johannes Triebs $^{1}$, and Jan Ole Hansen ${ }^{1 *}$ \\ ${ }^{1}$ R WTH A achen, Chair of Production Engineering of E-M obility Components, Campus B oulevard 30, \\ A achen, Germany
}

\begin{abstract}
Due to shorter product life-cycles, increasing product customization and the co-existence of electric and combustion engine vehicles, variant flexibility is gaining importance in the automobile production. The automobile body shop is characterized by inflexible, rigid fixture systems dedicated to meet the geometrical requirements of specific body parts. Changes in part geometry or dimension require the development of new fixture systems, thus increasing product variety results in higher fixture costs. This paper presents an approach for a fixtureless body shop based on component-integrated fixture-functions, increasing variant flexibility and reducing fixture costs. The approach is implemented using a body part assembly of an electric vehicle.
\end{abstract}

Keywords: automobile body shop, electric vehicles, fixtureless body shop, variant flexibility

\section{Introduction}

Automobile body shops are often rigid production systems with a high degree of automation for high production volumes. Due to its low flexibility and high investment costs in mid-digit million ranges [1], the body shop is particularly affected by increasing number of variants and shorter product life cycles. A significant part of the investment costs is caused by development and production of fixtures. According to Meichsner, variant-specific fixture costs can rise up to $40 \%$ of the total system costs in certain body shop stations [2]. Since fixtures are generally dedicated to meet the geometrical requirements of specific body parts, changes in part geometry or dimension, due to design changes between model years or due to integration of a new model to an existing production line, require the development and production of new fixtures. Consequently, the increasing product variety results in higher fixture costs.

Existing approaches in the body shop to flexibilize fixture are based mainly on the kinematisation of the fixture system whereby investment costs for kinematized fixture

* Corresponding author: j.hansen@pem.rwth-aachen.de 
systems can surpass those of conventional fixtures by $50 \%$. Further, flexibility gain of kinematized fixture systems is low due to limitations in adaptability and change time restrictions [3]. Therefore, research has increased efforts to replace fixture systems by an economic and flexibility increasing alternative technology. Feature-based fixturing approaches, enabling fixtureless body shop concepts by integrating fixture-functions within the body part through geometrical features, are expected to be advantageous in terms of investment costs savings and flexibility gain [4].

This paper describes a methodology to select and evaluate application-specific combinations of geometrical features and joining technologies suitable to replace conventional fixture systems. The approach is based on component-integrated fixturefunctions, which have been implemented in a fixtureless body shop station for an electric vehicle body part assembly using the described methodology.

\section{Practical approaches on fixture flexibilization}

In order to be able to produce cost-efficiently despite high initial investments and declining variant-specific quantities, body shops must be able to produce several vehicle variants flexibly on a single body shop line. By making body shops more flexible, investments in high-grade automation can be allocated to a larger number of products to be manufactured and justified economically. While the potential for flexibility in industrial robots can be exploited, for example, by changing tools and programs, fixtures that are specially adapted to specific part geometries considerably limit flexibility in automobile body shops. In recent years, increased efforts have been made to make the fixtures in body shops more flexible. The approaches implemented nowadays are mainly based on the kinematization of the fixture system, whereby the motion may concern the complete fixture (fixture changing systems) or be present within the fixture (e.g. adaptive clamping techniques).

Depending on the size of the fixture, the required kinematics are performed by industrial robots, rotary tables and drums or gantry systems and linear units (see Fig. 1) [5]. Small, lightweight fixtures are often stored in a fixture magazine and are replaced by industrial robots. Medium and large fixtures are mounted on rotary tables or drums, which are rotated to the body construction line depending on the vehicle variant to be manufactured. Despite the high total weight of up to $17,000 \mathrm{~kg}$ for table/drum and payload, the fixture change takes place within the cycle times. Due to the high space requirement, rotary tables and drums are suitable for accommodating two to four fixture variants. Rotary drum systems are used, for example, in the side frame production of the Audi Q5 [6]. The largest and heaviest fixtures weighing up to $2,500 \mathrm{~kg}$ are used in the framing stations of body shops, in which the base assembly, side frame and roof of the body are brought together. The vertical fixtures are mounted on gantry systems and linear units for transport to the body shop line.

The use of fixture change systems is suitable for a limited number of part variants and allows only limited flexibility in body shops. If the part geometry is changed, the complete fixture must still be replaced or an additional changing system must be integrated. Disadvantages are not only the costs for design and production, but also the space required, the associated storage and logistics costs as well as the capital commitment.

Compared to fixture changing systems, flexible fixtures use adaptable function carriers, whereby the clamping and determining elements adapt to different part geometries. On the one hand, the function carriers can be mounted on movable elements, which adapt to shape and size variations of the parts within given dimensions. The moving elements are often operated automatically via a pneumatic drive. In addition to movable function carriers, the clamping elements can be made more flexible by means of shape-variable adjustments. The clamping elements adapt to the part geometry by means of force or form-fit control, whereby pin cushions are often used in body shops [7]. The application flexibility of flexible 
fixtures is limited to just a few centimeters due to the adjustment ranges of the elements, consequently limiting the number of part variants. Further, investment costs in flexible fixtures often surpass those for conventional fixture systems and quality issues are common due to frequent adjustments and the elasticity of the systems. Thus, neither fixture changing systems nor flexible fixtures are economic viable solutions to increase flexibility in future automobile body shops.
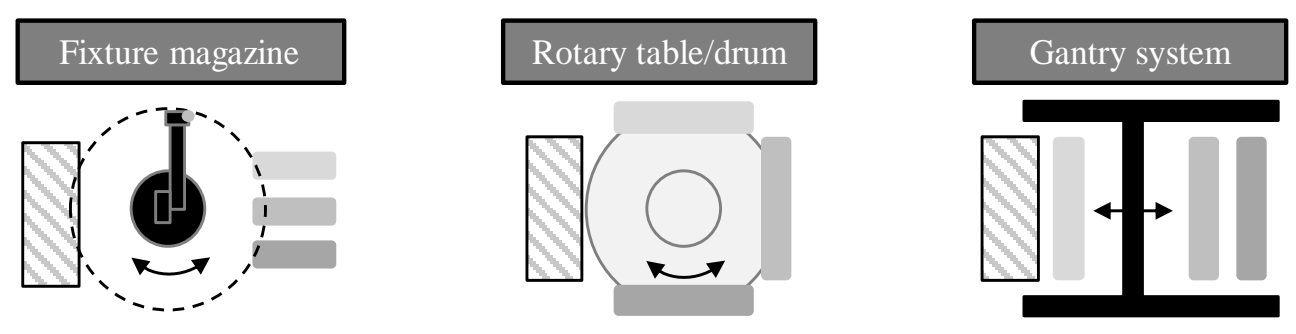

Fixture variant 1 Fixture variant 2 Fixture variant 3

Fig. 1. Concepts for fixture changing systems in automobile body shops.

\subsection{Literature review}

As mentioned above, a concept to reduce fixture costs and increase flexibility in a fixtureless body shop consists of relocating fixture-functions into the parts to be joined. In this concept, the fixture functions are taken over by geometrical features integrated into the parts. Therefore, approaches discussed following are related to the research area of feature-based fixturing (FBF). Adams and Whitney define these so-called 'assembly features' as objects that restrict one or more degrees of freedom between two parts to be joined and fix this restriction [8]. Naing supplements this description with the note that features used must always be considered as pairs, since the degrees of freedom constraint by a feature always depend on the mate [9].

When considering FBF approaches for body shop applications, several evaluation criteria should be taken into account according to Schlather [10]. Firstly, since geometrical feature are being integrated into the body parts, approaches should be applicable in early phases of the product development to ensure appropriate part design. Further, geometrical features should be considered to replace fixture and their functionalities, specifically positioning, clamping and fixing of body parts. A logic relating fixture-functions to geometrical features taking body shop tolerances and part accuracies into consideration should be presented. Considering the processes in automobile body shops, commonly used joining technologies such as spot welding or gluing should be considered when selecting features for specific use applications. In the following main approaches applicable to automotive body shops are discussed and evaluated regarding the described criteria listed in Table 1. 
Table 1. Criteria to evaluate FPF approaches for use in body shops.

\begin{tabular}{l}
\hline Evaluation Criteria \\
\hline Considers early phases of product development \\
Considers geometrical features to replace fixtures \\
Presents logic to relate geometrical features to fixtures-functions \\
Contemplates accuracy and tolerances in body shops \\
Considers joining technologies used in body shops \\
Describes feature selection process according to use-case
\end{tabular}

Koonmen developed a methodology called 'precision assembly technique' in 1994, which enables the assembly of components according to requirements from aircraft floor assemblies without fixtures. The technical concept is based on a combination of plug-in and mounting features. Together they can restrict a certain number of degrees of freedom before the joining operation. Further the author describes to what extent the entire assembly organization within a company would have to be adapted to the new concept and which extensive changes would be necessary for this [11]. The approach misses to describe the relation between fixture-functions and geometrical features. Further, neither accuracies and tolerances nor joining technologies utilized in body shops are considered.

In their approach to model assembly processes by pair characteristics, Chang and Perng focus on the development of capable feature-based part positioning mechanisms. To this end, characteristics and their characterizing properties (volumes, reference systems and interfaces) are described. Three types of combinations of features are outlined by the authors: fit-based, contact-based and orientation-based. Chang and Perng also examine the fastening of several superimposed parts and subdivide the assemblies into individual subassemblies [12]. Summarizing, the investigations focus less on descriptive or selection processes for geometrical features, but rather on the simplifications that can be achieved in the design phases for part positioning processes through the use of features.

Walczyk et al. developed a methodology for the fixture-free assembly of simple sheet metal parts for the aviation industry. The technical basis is tolerated alignment holes, which are drilled into the parts via CNC machines. At these alignment holes, the parts are connected to each other by temporary closures. On the basis of this part alignment and fixation, the parts are then finally joined at the points where the rivets or bolts are already positioned in the conventional case. Depending on the application, it may also make sense to use rivet or bolt holes already provided as alignment holes in order to avoid additional holes. To determine the optimum position and suitable tolerances of alignment holes, Walczyk et al. provide several indications, which refer in particular to the desired restriction of all degrees of freedom by the closures in interaction with the part geometry. The authors name so-called 'cleo clamps' as an exemplary temporary clamp lock, which expand after insertion into the holes and thus allow the parts to be centered and fixed. The clamps are removed after the joining process [13]. Nevertheless, no logic relating geometrical features to fixturefunctions is given by the authors. Further, neither accuracies and tolerances nor joining technologies utilized in body shops are considered.

Naing applies the FBF principle in the developed 'integrated methodology for jigless assembly' to the assembly of parts from the aviation industry. Naing explicitly includes the design phase in the methodology in order to integrate fixture functions into parts. The approach is divided into two main parts. Firstly, the author represents a mapping of all influences affecting the feature selection process. The influences are reflected time, cost and quality dimensions. However, it is pointed out that the cost dimension is a priority aspect and is related to all other influences. In a second part the author describes in detail 
the feature selection process as part of the methodology. He differentiates between location, support, clamping and fastening features for use in assembly processes. Based on this distinction, the author describes the selection process for positioning features in detail using examples from the aviation industry. For the selection of other characteristic types, general notes are given as to which influences should be taken into account in the selection. A feature library with characteristics for all four features types is created within the work. The characteristics library should be comprehensive, but no claim to completeness is made [9]. For the utilization in body shop applications, the approach misses considering joining technologies as well as the accuracies and tolerances arising in these processes.

With their principle of 'integral attachment', Messler et al. developed an approach for the mechanical fastening and joining of components by means of integrated attachment features. A firm connection is made by means of elastic, plastic or combined elastic-plastic deformation of the fastening features. The aim of integrated fastening is to implement fastening processes without additional tools, such as closures or fixtures. The main fastening features examined are snap-fits, in which an elastic plug feature is inserted into a fixed recording feature. On this basis, Messler et al. differentiate between positioning features ('locating features') and fixing features ('locking features'). 'Extended features' or 'Attributes' can also perform other supporting functions, such as improving userfriendliness. The authors also developed a classification scheme for various representative geometries of features and used this as a basis for investigating the combinability of different feature forms. The identified characteristics are divided into 'latches' and 'catches'. A combination matrix ('matrix of locking pairs') makes it possible to determine whether the respective plug-in and recording features can be combined with each other. A methodology consisting of six steps has been developed to facilitate the selection of appropriate fastening features. In doing so, all influences with regard to functions, arrangement, manufacture and robustness of features are taken into account. The selection of different fastening concepts is based mainly on a cost-based performance assessment [14]. Since the approach was developed for utilization in assembly processes, joining technologies in body shops are not taken into account. Further, the authors miss presenting a description of the feature selection process which considers accuracies and part tolerances.

Schlather developed a four-step methodology for the feature-based, fixtureless assembly of sheet metal structures, which was to address and improve various weak points of these based on an analysis of existing approaches. At first, the authors investigated and structured those factors that influence the dimensional accuracy and absorption potential of clamping and joining forces of feature-based fixture approaches. Based on this study, an approach for integrating the fixture functions into the parts was then developed in which the influencing factors are taken into account when selecting features. In a first step, suitable characteristic pairs for this approach are identified. Then characteristic pairs and parts are modeled and simulated to determine their influence on dependent variables. The core of the methodology follows with a detailed analysis of the interactions between features, parts and variables dependent on assembly and joining technologies. Concrete design guidelines for further implementation are derived from this analysis [10]. Nevertheless, neither a logic relating geometrical features to feature-functions nor a use-case specific feature selection process are presented.

By using FBF approaches, the use of conventional fixtures can be almost completely dispensed, resulting in corresponding investment reduction and flexibility gain. Nevertheless, the application of FBF approaches to automotive body shops presents further challenges which can be summarized in following points:

- Further research is needed to determine to what extent feature-based approaches are suitable for meeting the requirements specified in body shops, in particular with regard to tolerances and accuracies, 
- The influence of joining technologies relevant for automotive production on featurebased approaches has not yet been taken into account,

- There are no guidelines for designers in early development phases on how to select geometrical features for specific use cases taking the above points into account.

The points are to be supplemented by the lack of inclusion of methodological considerations in the design of the respective features. Thus, the identified research demand forms the central motivation of the approach presented following.

\section{Approach to select geometrical features for fixtureless body shops}

Since the approach is to be understood as part of an early stage development process, its procedure must be based on a theoretically founded design methodology. In this case, the Pahl/Beitz design methodology is used as a basis [15]. The proposed approach comprises a total of four steps (methods), each of which is used to develop an overall methodology using suitable tools. Each method of the overall methodology generates output variables at product and data level, taking into account the input variables at product and data level and the associated methods at method level (see Fig. 2). Basis for the methodology is a model of the considered system, in this case a product, and the associated framework (replacing fixture in body shop), which is used as an input variable and then further developed in the course of the methodology.

Therefore, the methodology is initially provided with a 'model of the framework' as a mapping of the application case with all data relevant to the methodology. By applying method I, these data is systematically mapped for further use in the methodology. In the following steps, internal data generated in the previous methods is used in addition to relevant external data supplied. All data systematized and generated in the methodology is systematically presented in the form of a 'product development sheet' and made available for further use in the development process.

The first step of the methodology takes up the model of the framework and prepares it for further analysis. An essential component of method I is to depict the considered joining points in the 'effector system' of the fixtures used in the production process. The effector system for fixtures was introduced by Trummer and Wiebach [16] and is used to systematically record and categorize the multitude of influences within fixture-related processes. The basic idea is that the considered joining points with integrated geometrical features take up the place of fixture within conventional body shop and must accordingly take into account all influences acting there. Therefore, the metal sheets to be joined in the body shop station are abstracted to their joining points. Joining points are described by means of the type of joint between the parts, in general parallel-joint or line-joint. Thus, the output of the method are the considered joining points in a transformed effort system for fixtures in the body shop.

Input data
Illustration of
the application
case by means
of a model of
the framework
with all
relevant
associated data

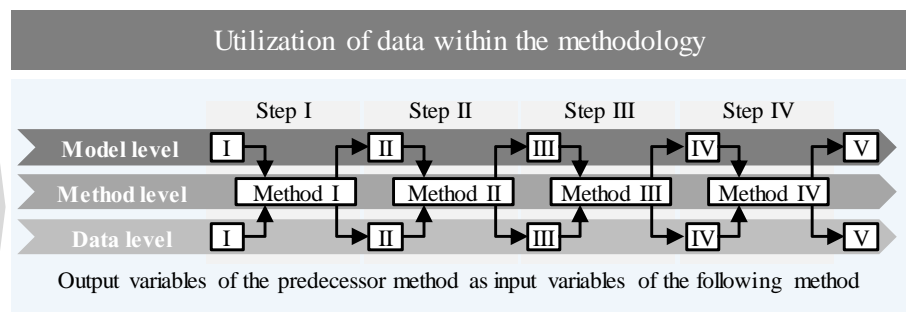
Output data Product development sheet for mapping the generated data for further use in the development process

Fig. 2. Input-Output relations between methods and levels of the methodology. 
The requirements for the joining point can be derived from this transformed effort system for step 2 of the methodology. These requirements are divided into requirements derived from fixture-functions and further original requirements that have to be considered for joining points. The derived requirements with their characteristics are presented in a morphological box. Since the features to be selected are to take over the functions of a fixture in conventional body shops, it must first be derived how the fixture functions can be recorded and which associated characteristics can occur. The identified requirements thus reflect the role of the fixtures in the original effort system or the role of the joining points in the transformed effort system.

The fixture-function positioning is represented in the methodology from the perspective of relative assembly time and centering. The relative assembly time takes into account the cycle times specified in production planning. On this basis, the portion of the cycle time available for the positioning of the metal sheets within the overall process is determined. Centering describes the location of the parts relative to each other. By centering the assembly through geometrical features integrated into the parts, tolerances and accuracies inherent by the features are considered. Production-related tolerances and accuracies of the features can be described by knowledge of their manufacturing processes. The fixture-functions clamping and fixing are represented by the number of degrees of freedom to be restricted per direction of movement before the joining process. Accordingly, three translatory and three rotational degrees of freedom must be taken into account, each of which must be restricted in none, only in positive, only in negative or in both coordinate directions. In most applications it is generally desirable to restrict all degrees of freedom. However, there are cases in which a fixing in one coordinate direction is explicitly not desired. This may be the case, for example, if thermal expansion due to joining processes follow and the degrees of freedom must remain unrestricted. The size and exact geometry as well as the number of features to be selected determine whether the parts remain safely in the determining position under the influence of the joining process. The three elements of fixing forces, magnitude, direction and point of application, are considered in this work.

The feature assignment and selection processes in steps 3 and 4 of the methodology require describing all possible eligible geometrical features, taking into consideration the constraints and degrees of freedom resulting from joining technologies. As shown in Fig. 3, the relation between part integrated geometrical features, joining processes as well as the joining point requirements derived in the second step of the methodology are therefore considered within multi-domain matrices (MDM). According to Maurer [17], an indefinite number of domains can be analyzed within MDM matrices, as matrix entries are themselves matrices.
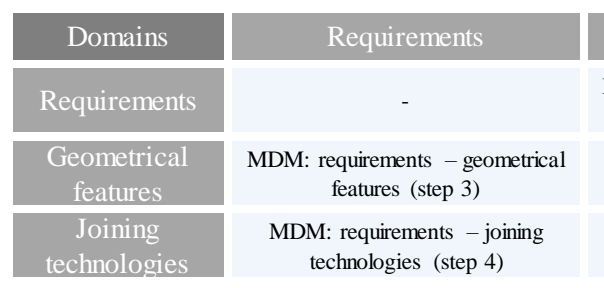

Geometrical features
MDM : requirements - geometrical
features (step 3)
MDM : geometrical features -
joining technologies (step 4)

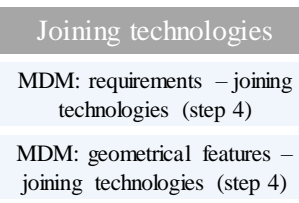

Fig. 3. Requirements, features and joining technologies are related in multi-domain-matrices (MDM).

Feature assignment, as the third step in the methodology, describes the relation between the requirements derived in step 2 with all eligible geometrical features, depending on their degree of requirement fulfillment. For this purpose, an abstracted description of geometrical features is required in order to structure and classify the large amount of possible features. Feature characteristics differ, among others, in regard to their geometry, material and 
assembly process. The authors therefore propose to describe features by geometrical (e.g. ball), functional (e.g. undercut) and process related (e.g. fixing force) factors. Geometrical characteristics of the features are outlined by formulas, as e.g. volume or surface of a ball can easily be calculated. From the geometry of the feature, a functional characteristic is derived. The functional characteristic then defines a requirement fulfillment within the process of geometry setting between the parts to be joined. A feature with an undercut structure would, for example, constraint more degrees of freedom than an overlaying feature due to the fixing force it applies to the mate sheet (see Fig. 4). Therefore, it can occur that degrees of freedom are over-determined, which leads to constraints regarding certain featurefeature combinations.

For the final selection of geometrical features, the constraints and degrees of freedom resulting from the influence of joining technologies need to be considered. Certain combinations of geometrical feature and joining technology can meet requirements the feature alone would not meet. For example: if waterproofness is a joining point requirement and the pre-selected feature a hole or slot, which initially would not meet the requirement, the feature could be sealed by the joining technology in case of laser welding or gluing. Therefore, new degrees of freedom can be generated through the utilization of certain joining technologies. On the other hand, there are geometrical (e.g. unilateral accessibility), functional (e.g. heat input) or company related (e.g. joining guidelines) limitations towards the utilization of specific joining technology and feature combinations. All possible featurejoining technology combinations and their requirement fulfillment are outlined within the multi-domain-matrices used to describe the relations between these domains. Positive relations (additional degrees of freedom) are assigned accordingly, while negative relations (additional constraints) are disqualified within the matrix.

The eligible combinations result in a reduced selection of suitable features and joining technologies. From this selection, the feature amount and type is determined. Criteria for the final feature-joining technology combination selection comprise economic factors. Thus, the investment costs for conventional fixture systems have to be compared with the costs of feature integration, as well as costs for the respectively utilized joining technologies. Criteria regarding body quality and process stability are included in the requirements derived in the second step of the methodology and therefore being taken into account in steps 3 and 4 .
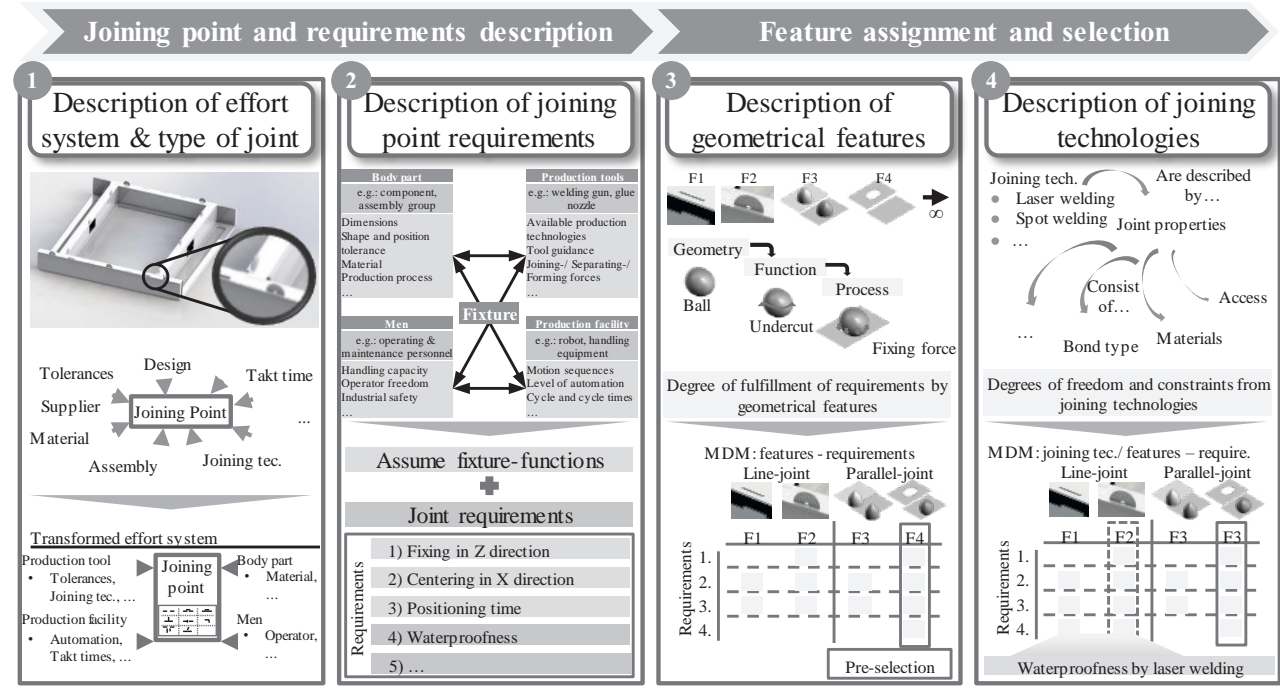

Fig. 4. Methodology to select geometrical features for a fixtureless body shop. 


\section{Implementation}

The methodology to select geometrical features and joining technologies has been applied for a fixtureless body shop station of an electric vehicle body part. In the reference body station three body parts are joined, whereby the station contains several fixtures for part positioning and clamping as well as spot welding and transport robots for fixing and transportation of the assembly ${ }^{\dagger}$. For this work the original body parts were redesigned incl. geometrical features for positioning and fixing based on the reference requirements regarding parts and processes. Further, joining technologies for the pre-selected features were analyzed and selected. Finally, two combinations of geometrical features and joining technologies fulfilled all requirements and were considered besides the use of conventional fixture systems. Both features show an undercut structure and therefore consider fixing of the parts. While one feature is joined using conventional spot welding, the other feature is combined with laser welding due to accessibility constraints.

All three scenarios (conventional fixtures, undercut feature-spot welding as well as undercut feature-laser welding) were afterwards analyzed in regard to their economic potential, taking into account investment costs for the fixture, costs for the production and part integration of the features, costs for joining as well as the cycle time. Overall cost reductions amounted to $18 \%$ and $31 \%$ respectively for feature-spot welding and feature-laser welding combinations in comparison to the conventional fixture system. Cycle time savings of up to $30 \%$ were also realized. Further, the fixtureless options increase flexibility, as otherwise the integration of an additional variant would have cost a mid-6-digit investment amount due to new fixtures and other adaptions necessary.

\section{Conclusions and future work}

In this paper, a methodology to select geometrical features and related joining technologies to realize a fixtureless body shop concept is outlined. The methodology is based on a four step approach to describe requirements and joining points as well as assign and select features and joining technologies. Ongoing research on the methodology comprises further detailing of the process steps and further practical realization on body shop station in larger scale. Generating more data in practical implementations will allow detailing the assessment of the methodologies' advantage regarding investment cost reduction and flexibility gain.

\section{References}

1. S. Spieckermann, K. Gutenschwager, H. Heinzel, S. Voß, Simulation-B ased Optimization in the A utomotive Industry, Simulation 75(5), 276-286 (2000)

2. T. M eichsner, Migrationskonzept für einen modell- und variantenflexiblen Automobilkarosseriebau, PhD Thesis (Universität Hannover, Hannover, 2007)

3. T. Haunstetter, Methoden des Data Mining in Anwendung an die Flexibilitätsattribute des automobilen Karosseriebaus zur strategischen Investitionsplanung, PhD Thesis (Universität der Bundeswehr Hamburg, Hamburg, 2010)

4. J. Hansen, A. Kampker, J. Triebs, Methodology for flexibility in the future automobile body shop: results of a comprehensive cross-industry study (Presentation CIR P-CM S, Stockholm, 2018)

\footnotetext{
$\lceil$ All values and numbers have been adjusted or are being omitted as not to allow any conclusions to be drawn about the company regarded.
} 
5. J. A bulawi, Beherrschung der Komplexität von 3D-CAD-Modellen, PhD Thesis (Universität der Bundeswehr Hamburg, Hamburg, 2012)

6. O. Schol, Der neue Audi Q5: Entwicklung und Technik (ATZ/M TZ-Typenbuch, V ieweg+T eubner, V erlag, 2009)

7. M. Franzkowiak, Methodik zur Strukturierung von Vorrichtungssystemen in der Lohnfertigung, PhD Thesis (Technische Universität München, München, 2013)

8. J. A dams, D. Whitney, A pplication of Screw Theory to Constraint A nalysis of M echnical A ssemblies J oined by Features, J. M ech. Des. 123(1), 26-32 (2001)

9. S. Naing, Feature Based Design for Jigless Assembly, PhD Thesis (Cranfield University, Cranfield, 2004)

10. F. Schlather, F. O efele, M. Zeah, T oward a feature-based approach for fixtureless build-up of sheet metal structures, J. Eng. Technol. Res. 5(4), 97-102 (2016)

11. P. Koonmen, Implementing precision assembly techniques in the commercial aircraft industry, M aster Thesis (M assachusetts Institute of Technology, M assachusetts, 1994)

12. C.F. Chang, D.B. Perng, A ssembly-part automatic positioning using high-level entities of mating features, Comput. Integr. M anuf. Syst. 10(3), 205-215 (1997)

13. D.F. Wal czyk, V. Raju, R. M iller, Fixtureless assembly of sheet metal parts for the aircraft industry, in Proceedings of the Institution of M echanical Engineers Part $B$ Journal of Engineering M anufacture, 214(3), 173-182 (2000)

14. R. M essler, Integral Mechanical Attachment (Butterworth-H einemann, Oxford, 2006)

15. J. Feldhusen, K.H. Grote, Der Produktentstehungsprozess (PEP). In J. Feldhusen, K.H. Grote (Eds.), Pahl/Beitz Konstruktionslehre (Springer V ieweg, Heidelberg, 2013)

16. A. Trummer, H. W iebach, Vorrichtungen in der Produktionstechnik (V iewegs Fachbücher der Technik, Springer Fachmedien, Wiesbaden, 1994)

17. M. M aurer, Structural Awareness in Complex Product Design, PhD Thesis, (Technische Universität München, München, 2007) 